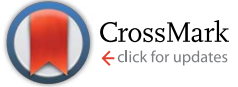

Cite this: RSC Adv., 2017, 7, 9064

Received 26th October 2016 Accepted 20th January 2017

DOI: $10.1039 / c 6 r a 25888 c$

rsc.li/rsc-advances

\section{Wetting characteristics of underwater micro-patterned surfaces $\uparrow$}

\author{
Surjyasish Mitra, Naga Siva Kumar Gunda and Sushanta K. Mitra*
}

\section{Introduction}

Micro and nano-textured hierarchical structures have found applications in self cleaning windows, ${ }^{1}$ systems where protection from marine bio-fouling is required, ${ }^{2}$ biomimetics ${ }^{3-7}$ as well as surfaces producing low drag in fluid flow. ${ }^{8,9}$ The theoretical basis of such behavior is attributed to the superhydrophobic or super-oleophobic nature of such textured surfaces, governed by the well-known Wenzel ${ }^{10}$ and CassieBaxter ${ }^{11}$ configurations. When a liquid drop is deposited on a rough (or textured) substrate kept in ambient air medium, a Wenzel state corresponds to the drop penetrating the gap between the micro structures resulting in complete wetting, while a Cassie-Baxter state corresponds to the drop hanging on top of the micro structures with a layer of air trapped beneath (see Fig. 1). The validity of these two wetting theories is often debated and a cautious approach to use them is proposed in many recent studies. ${ }^{12-23}$ The differences mostly stem from the fact that these wetting theories have proved to be insufficient in characterizing contact angle hysteresis (i.e., the range of contact angles exhibited by a liquid drop on a given rough substrate), which is crucial towards understanding of hydrophobicity (or superhydrophobicity).

Micro \& Nano-scale Transport Laboratory, Department of Mechanical Engineering, Lassonde School of Engineering, York University, Toronto, Ontario M3J 1P3, Canada. E-mail: sushanta.mitra@lassonde.yorku.ca; Tel: +1 4167365924

$\dagger$ Electronic supplementary information (ESI) available. See DOI: $10.1039 /$ c6ra25888c
Studies by Extrand et al. ${ }^{17,21}$ Gao et al. ${ }^{12,22,23}$ and others have stressed that the interfacial contact areas are less significant for the wetting configuration of a drop on a rough (heterogeneous or textured) substrate. ${ }^{24-30}$ Rather, interaction of the threephases in the vicinity of the three-phase contact line (i.e., the drop-substrate-air contact line) mostly dictates wetting configuration. Alternate models have been proposed in recent times to explain wetting behavior on such surfaces, especially to explain the discrepancies between experimentally observed apparent advancing and receding contact angles (i.e., contact angle hysteresis $\left.{ }^{31}\right)^{17,19,26-29,32-36}$ with those predicted by Wenzel and Cassie-Baxter theories. Still, for the majority of the reported studies involving wetting on rough (or textured) surfaces, the conventional form of Wenzel and Cassie-Baxter theories have proved to be successful in describing experimental data. ${ }^{1,2,8,14-16,18,37-42}$ In a recent work involving the wettability of chemically textured copper slabs having multi-tier roughness, Frankiewicz and Attinger suggested that there could be a possibility of meta-stable Cassie-Baxter configuration and even observed reversible wetting transitions between Wenzel and Cassie-Baxter wetting states. ${ }^{43}$

Another prominent issue regarding the applicability of these wetting theories is that they are solely based on interfacial area fractions and do not provide adequate clarity related to the transition from a Cassie state to a Wenzel state. Even though a Wenzel state corresponds to a lower energy state for the drop, during the wetting process, often the drop remains in a Cassie state due to a large energy barrier associated with making a transition between the two states. ${ }^{40}$ In such cases, some 


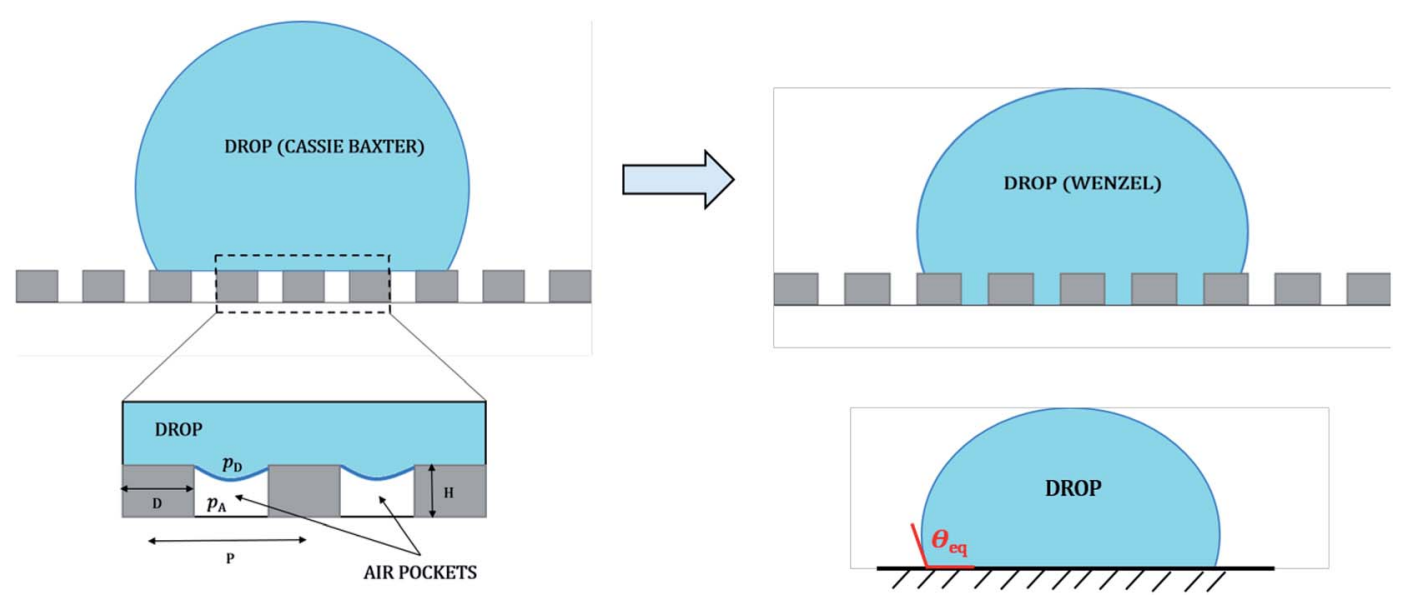

Fig. 1 Schematic of a liquid drop undergoing a transition from a Cassie-Baxter to Wenzel state on a micro-patterned substrate (with pillar width $D$, height $H$ and pitch $P$ ) in air. The enlarged view shows the drop-air interface hanging between adjacent pillars. $p_{D}$ and $p_{\mathrm{A}}$ are the drop and surrounding air pressure, respectively. The configuration of the drop on the corresponding flat substrate (with equilibrium contact angle $\theta_{\text {eq }}$ ) is also shown for reference.

external work needs to be done to make the transition. ${ }^{40,44,45}$ Possibility of multiple equilibrium states ${ }^{40,46}$ on the same substrate often makes it difficult to decide which of the two wetting theories is to be used for the effective design of superhydrophobic surfaces. Efforts have been made to reconcile this debatable issue of wetting transition and provide a better understanding of the energy barrier associated with such transitions. ${ }^{45,47-54}$ Such an energy barrier is often expressed in terms of a critical pressure ${ }^{48,49}$ that exists across the drop-air interface in the gap between any two adjacent pillars, which ensures whether the Cassie-Baxter to Wenzel transition is favorable or not (see Fig. 1). Mathematically the transition condition in terms of liquid pressure can be expressed approximately as ${ }^{48,49}$

$$
p_{\mathrm{D}}-p_{\mathrm{A}}>-\frac{2 \gamma_{\mathrm{DA}} \cos \theta_{\mathrm{eq}}}{(P-D)}
$$

where, $p_{\mathrm{D}}$ and $p_{\mathrm{A}}$ are the drop and surrounding air pressure, respectively; $P$ and $D$ are the pillar pitch and width (or diameter), respectively; $\gamma_{\mathrm{DA}}$ is the surface tension of liquid drop in air and $\theta_{\mathrm{eq}}$ is the equilibrium contact angle of the liquid drop on the corresponding flat substrate. If the above condition is satisfied, there will be a transition of the drop to a Wenzel state. Otherwise, the drop will be in a (meta)stable Cassie-Baxter state. ${ }^{48,49}$

Even though wetting characteristics of textured surfaces kept in ambient air medium have been extensively studied, there is a significant lacuna towards understanding of wetting characteristics of such textured surfaces kept underwater (or underliquid). ${ }^{55-59}$ We can look towards nature where fish scales and shark skins have hierarchical structures enabling them to repel oil drops and other contaminants from their surfaces. Hence, a study involving behavior of oil drops on an underwater substrate mimicking such phenomenon observed in nature finds applications in designing surfaces like submerged ship parts, marine pipelines, etc., which can effectively repel oil drops in case of an oil spill. ${ }^{60-62}$ The question remains is that whether Cassie-Baxter or Wenzel formulation is adequate to characterize wetting for under-liquid micro-structured surfaces? Most hydrophilic surfaces in air exhibit oleophobicity when placed underwater, i.e., the oil drop in its equilibrium configuration shows an underwater contact angle greater than $90^{\circ} .{ }^{55}$ It is the oil-water-solid contact angle which is the focus here, pertinent to a micro-patterned surface. In other words, we aim to check whether the Wenzel and Cassie-Baxter theories hold true for such underwater micro-patterned surfaces. If not, is there a need to have alternate considerations like the analysis of free energy barrier while studying the wetting of such under-liquid surfaces? Also, of interest here is to study the hysteresis of oil drops on such surfaces. Hence, to provide meaningful answers to these new questions related to under-liquid wettability, we have fabricated silicon based micro pillared substrates with a regular square array of cylindrical pillars having constant pillar height and diameter but varying pillar spacing. The substrates were placed underwater and wetting characteristics of millimeter sized oil droplets were studied in terms of their static (or equilibrium), advancing and receding contact angles.

\section{Experimental section}

\subsection{Sample preparation}

In this work, we have chosen the simplest and widely used micro-fabricated structure, which is the pillar geometry. The cost of fabrication of such pillared chip is around CAD 10 (based on academic pricing at University of Toronto micro/nanofabrication facilities). Any other shape would incur huge expense and also additional microfabrication steps. Hence, we have limited our study to this base case with pillar geometry. A $100 \mathrm{~mm}$ diameter silicon (Si) substrate (Wafer World Inc., West Palm Beach, FL, USA) was cleaned in a standard piranha solution $\left(\mathrm{H}_{2} \mathrm{SO}_{4}\right.$ and $\mathrm{H}_{2} \mathrm{O}_{2}$ in $3: 1$ ratio), then rinsed with deionised (DI) water, and dried with nitrogen gas. A positive photoresist (PPR) S1818 (MicroChem Corp, Westborough, MA, USA) was 
spin coated at $3000 \mathrm{rpm}$ for 30 seconds and the coated substrate was soft baked at $115{ }^{\circ} \mathrm{C}$ for 60 seconds. The pillars were patterned on PPR with standard photolithography by exposing the substrate at $100 \mathrm{~mJ} \mathrm{~cm} \mathrm{~cm}^{-2}$ and then developing the substrate in MF-319 developer (Rohm and Haas Electronic Materials LLC, Marlborough, MA, USA). Further, the silicon substrate is etched anisotropically using deep reactive ion etching (DRIE) method for about $15 \mu \mathrm{m}$. After etching, the photoresist was removed by placing the substrate in PRS-100 stripper (HTA Enterprises, Microchrome Technology Products, San Jose, CA, USA). The substrates were then cut into $6 \times 6 \mathrm{~mm}^{2}$ pieces. Using this process, substrates with constant pillar diameter $50 \mu \mathrm{m}$ and height $15 \mu \mathrm{m}$ but varying pitch $(60 \mu \mathrm{m}, 75 \mu \mathrm{m}$ and $100 \mu \mathrm{m}$, respectively) were fabricated (see Fig. 2). The complete fabrication process flow for this type of pillar structures on a silicon substrate can be found elsewhere. ${ }^{63-65}$ We have also used one additional $100 \mathrm{~mm}$-diameter silicon wafer and performed all the chemical treatments, outlined earlier, except the patterning process. Such wafer was also diced into $6 \times 6 \mathrm{~mm}^{2}$ pieces, which were then used as standard reference substrates for contact angle measurements.

\subsection{Instrumentation}

For the experimental setup, a custom made contact angle measurement system was used. The working liquids used were deionized water (density $\rho_{\mathrm{W}}=1000 \mathrm{~kg} \mathrm{~m}^{-3}$, surface tension in air $\gamma_{\mathrm{WA}}=72.1 \mathrm{mN} \mathrm{m}^{-1}$ ) and laser oil (Cargille Laboratories Inc., Cedar Grove, NJ, USA; density $\rho_{\mathrm{O}}=1100 \mathrm{~kg} \mathrm{~m}^{-3}$, surface tension in air $\gamma_{\mathrm{OA}}=24.5 \mathrm{mN} \mathrm{m}^{-1}$ ). The oil-water interfacial tension, $\gamma_{\text {ow }}$ is $33.33 \mathrm{mN} .{ }^{66}$ For static contact angle measurements, the substrates were kept at the bottom of a distortion free glass cuvette (Krüss Germany, SC-01) filled with DI water and millimeter sized oil drops were deposited. Care was taken to ensure that no entrapped air was present in between the pillars when the substrates were submerged in water. This was evident from the absence of any bubble within the bulk water phase during the course of experiment. For each set of experiment, the substrates were submerged for $5 \mathrm{~min}$, thereby preventing any diffusion of entrapped air (if any) into the water phase.

The images were captured using a CCD camera and analyzed using an in-house developed Drop Shape Analysis software. A tangent method $^{67}$ was used to extract the contact angle information from the observed slope of the three phase contact line associated with each image of the sessile drop (see Fig. 3). For hysteresis measurements, the needle tip used for drop deposition was inserted into an already deposited sessile drop and the syringe pump associated with the needle-drop assembly of the
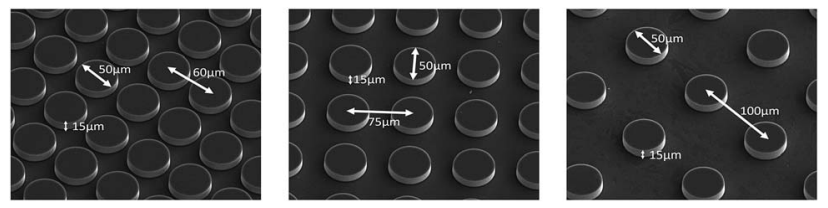

Fig. 2 SEM micrographs of the Si micro-patterned substrates with the different pillar configurations.

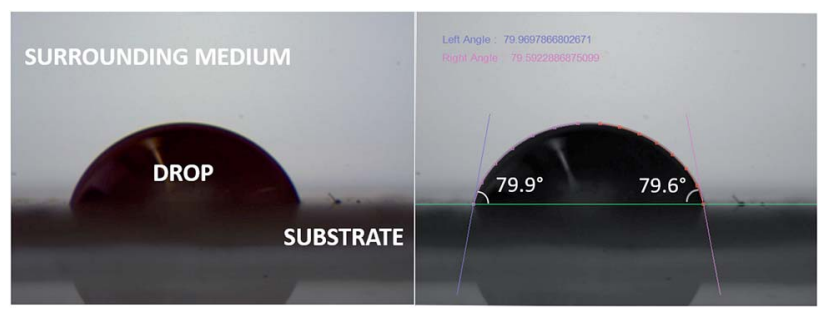

Fig. 3 Extraction of contact angle from obtained images of oil/water drop on the given substrate (micro-patterned or flat) kept underwater or in air using the tangent method.

contact angle measurement system was operated at a flow rate of $10 \mu \mathrm{L} \mathrm{min}{ }^{-1}$ to increase (or decrease) the drop volume. ${ }^{67}$ It is to be noted that in the original experiments of Wenzel, ${ }^{\mathbf{1 0}}$ a quasi-equilibrium condition was maintained by gently moving the plate. In this current set-up, as reported in our earlier work, ${ }^{67}$ we ensure the quasi-equilibrium process for the oil-water-solid system by controlling the flow rate (injection and retraction) of the needle-drop assembly during the hysteresis measurements. As the drop volume was increased, the contact angle reached a maximum value close to $180^{\circ}$ and on further increase, the three phase contact line jumped on to the next pillar. This maximum contact angle, i.e., the advancing contact angle, $\theta_{\mathrm{A}}$ was calculated from the recorded images. In a similar manner, when liquid was withdrawn from the drop by the needle, the receding contact angle, $\theta_{\mathrm{R}}$ was measured. For every micro-pillar configuration, each set of contact angle measurements (advancing, receding and equilibrium) was repeated 5 times to ensure consistency. The contact angle values provided here are the average values of 5 measurements and are reproducible within an experimental error of $\pm 2^{\circ}$. Contact angle measurements were also conducted on the standard reference flat Si substrates, described earlier, for comparison purpose.

\section{Results}

\subsection{Static contact angles}

Fig. 4A and B show the static contact angles of oil in air, water in air and oil in underwater systems for the micro-patterned and flat silicon substrates, respectively. For an oil drop on a flat solid surface placed underwater, the equilibrium contact angle, $\theta_{\mathrm{OW}}$ can be evaluated by applying the Young's equation for water (drop)-air, oil (drop)-air, and oil (drop)-water systems, respectively as, ${ }^{55}$

$$
\cos \theta_{\mathrm{OW}}=\frac{\gamma_{\mathrm{OA}} \cos \theta_{\mathrm{OA}}-\gamma_{\mathrm{WA}} \cos \theta_{\mathrm{WA}}}{\gamma_{\mathrm{OW}}}
$$

where $\gamma_{\mathrm{OA}}, \gamma_{\mathrm{WA}}$ and $\gamma_{\mathrm{OW}}$ are the oil-air, water-air and oil-water interfacial tensions, respectively. $\theta_{\mathrm{OA}}$ and $\theta_{\mathrm{WA}}$ are the equilibrium contact angles of oil and water drops on a flat substrate in air, respectively. For the present case, with micro-patterned surfaces consisting of a regular square array of cylindrical pillars with diameter $D$, height $H$ and pitch $P$, the Wenzel and Cassie-Baxter configurations of a liquid drop on such surfaces can be denoted by: ${ }^{55}$ 

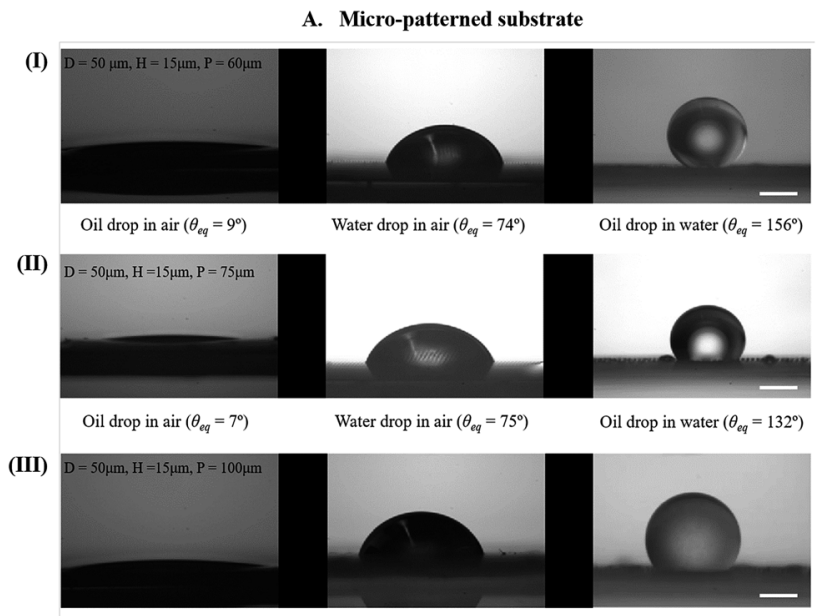

Oil drop in air $\left(\theta_{e q}=4^{\circ}\right) \quad$ Water drop in air $\left(\theta_{e q}=80^{\circ}\right) \quad$ Oil drop in water $\left(\theta_{e q}=117^{\circ}\right)$

B. Reference flat surface

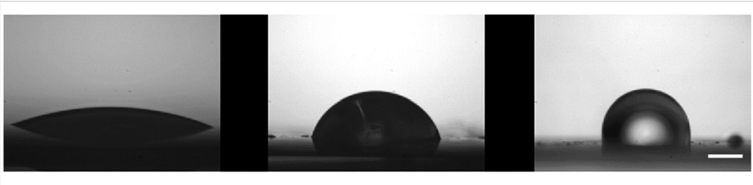

Oil drop in air $\left(\theta_{e q}=22^{\circ}\right) \quad$ Water drop in air $\left(\theta_{e q}=80^{\circ}\right) \quad$ Oil drop in water $\left(\theta_{e q}=86^{\circ}\right)$

Fig. 4 (A) Static contact angle measurements of oil-Si-air, water-Siair and oil-Si-water systems for the different micro-patterned silicon substrates: (I) $D=50 \mu \mathrm{m}, H=15 \mu \mathrm{m}, P=60 \mu \mathrm{m}$, (II) $D=50 \mu \mathrm{m}, H=15$ $\mu \mathrm{m}, P=75 \mu \mathrm{m}$ and (III) $D=50 \mu \mathrm{m}, H=15 \mu \mathrm{m}, P=100 \mu \mathrm{m}$. (B) Equilibrium configurations of oil-Si-air, water-Si-air and oil-Siwater systems for the reference flat silicon substrate. The scale bar represents $1 \mathrm{~mm}$.

$$
\begin{aligned}
& \cos \theta_{\text {Wenzel }}=\left(1+\frac{\pi D H}{P^{2}}\right) \cos \theta_{\mathrm{eq}} \\
& \cos \theta_{\mathrm{CB}}=\frac{\pi D^{2}}{4 P^{2}}\left(\cos \theta_{\mathrm{eq}}+1\right)-1
\end{aligned}
$$

where $\theta_{\mathrm{eq}}\left(\theta_{\mathrm{OA}}, \theta_{\mathrm{WA}}, \theta_{\mathrm{OW}}\right)$ is the equilibrium contact angle on a flat surface. It can be seen that all different micro-patterned substrates exhibit oleophobicity when placed under water.

Table 1 compares the equilibrium contact angles measured from experiment with the theoretical Wenzel values for cases where the measurements were conducted in air. It can be concluded that for wetting in air, the water drop exhibited a Wenzel configuration for all cases, while oil exhibited

Table 1 Comparison of static contact angles of water drops on the micro-patterned surfaces in air with those predicted by Wenzel configuration (i.e., by computing $\theta_{\text {Wenzel }}$ from eqn (3) using experimentally observed equilibrium contact angle $\theta_{\mathrm{WA}}$ )

\begin{tabular}{lll}
\hline Pitch $(\mu \mathrm{m})$ & $\begin{array}{l}\text { Observed equilibrium } \\
\text { CA of water in air }\end{array}$ & $\begin{array}{l}\text { Theoretical equilibrium } \\
\text { CA of water in air }\end{array}$ \\
\hline 0 (flat) & $80^{\circ}\left(\theta_{\mathrm{WA}}\right) \pm 1^{\circ}$ & - \\
60 & $74^{\circ} \pm 1^{\circ}$ & $73^{\circ}$ \\
75 & $75^{\circ} \pm 1^{\circ}$ & $75^{\circ}$ \\
100 & $80^{\circ} \pm 1^{\circ}$ & $77^{\circ}$
\end{tabular}

complete wetting with very low contact angles (see the first column from the left in Fig. 4A). But in case of wetting of oil drops in underwater configuration, neither the Wenzel nor the Cassie-Baxter theory was in accordance with the observed static contact angle values (see Table 2).

It can thus be concluded that as far as static configurations are concerned, the Wenzel (or Cassie-Baxter) theory does not accurately capture the wetting dynamics for underwater systems. Jung et al., ${ }^{55}$ in their recent study of wetting on underwater micro-patterned substrates, which consists of pillars of constant height and diameter of $30 \mu \mathrm{m}$ and $14 \mu \mathrm{m}$, respectively, with the pillar pitch varied from $21 \mu \mathrm{m}$ to $210 \mu \mathrm{m}$, found that Wenzel and Cassie-Baxter theories match well with their experimental data. In their experiments, they have used hexadecane as the liquid drop $(R=1 \mathrm{~mm})$ and water as surrounding liquid medium and found that the equilibrium contact angles for under-liquid micro-patterned silicon substrates varied from $146^{\circ}$ for the $21 \mu \mathrm{m}$ pitch substrate (Cassie-Baxter) to $104^{\circ}$ for the $210 \mu \mathrm{m}$ pitch substrate (Wenzel). However, no contact angle hysteresis analysis was performed by Jung et al. This mismatch in the equilibrium contact angle value for a drop on an under-liquid substrate between our present study and those provided by Jung et al. ${ }^{55}$ has propelled us to perform a hysteresis study to gather more insight into this wetting problem.

\subsection{Contact angle hysteresis}

Fig. 5(i) shows the advancing contact angles for different micropatterned surfaces used. It should be noted here that capturing of images with drop on micro-patterned substrate kept in underwater is a challenge. The pillars are not completely visible from the obtained images (see (a) and (b) of Fig. 5(i)). Only after significant image analysis, which includes changing the image contrast, the pillars become gradually visible (see (c) of Fig. 5(i)) and the three-phase contact line (TPCL) is correctly identified. For all the micro-patterned substrates, the advancing contact angle is close to $180^{\circ}$. The difference in obtained contact angle values can be attributed to the difference in image clarity and hence a slight deviation in the obtained fitting while computing the contact angle using the tangent method. To keep things in perspective, one can compare these values with the more wellstudied system of contact angle hysteresis in an ambient air medium. ${ }^{\mathbf{1 4 , 1 7 , 1 9 , 2 0 , 4 1 , 6 8}}$ For cases where the substrate is kept in air

Table 2 Comparison of static contact angles (CAs) of oil drops on the underwater micro-patterned surfaces with those predicted by Wenzel (eqn (3)) and Cassie-Baxter (eqn (4)) configurations (i.e., by computing $\theta_{\text {Wenzel }}$ and $\theta_{\mathrm{CB}}$ from eqn (3) and (4), respectively, using experimentally observed static contact angle $\theta_{\mathrm{OW}}$ )

\begin{tabular}{llll}
\hline Pitch $(\mu \mathrm{m})$ & $\begin{array}{l}\text { Observed CA } \\
\text { of oil in water }\end{array}$ & $\begin{array}{l}\text { Wenzel CA of } \\
\text { oil in water }\end{array}$ & $\begin{array}{l}\text { Cassie-Baxter CA } \\
\text { of oil in water }\end{array}$ \\
\hline 0 (flat) & $86^{\circ}\left(\theta_{\text {Ow }}\right) \pm 1^{\circ}$ & - & - \\
60 & $156^{\circ} \pm 1^{\circ}$ & $86.6^{\circ}$ & $115^{\circ}$ \\
75 & $132^{\circ} \pm 1^{\circ}$ & $87^{\circ}$ & $129^{\circ}$ \\
100 & $117^{\circ} \pm 1^{\circ}$ & $87.5^{\circ}$ & $142^{\circ}$
\end{tabular}




\section{i. Advancing Contact Angle}

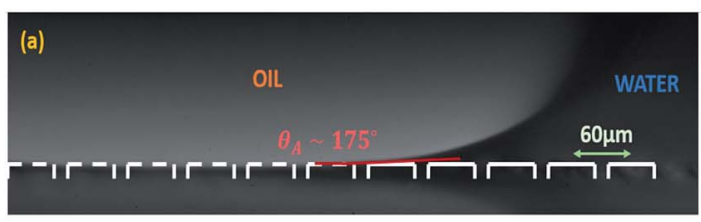

$D=50 \mu \mathrm{m}, \mathrm{H}=15 \mu \mathrm{m}, \mathrm{P}=60 \mu \mathrm{m}$

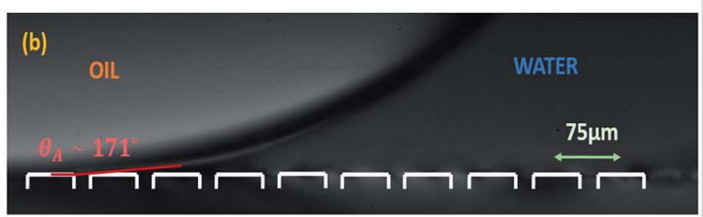

$\mathrm{D}=50 \mu \mathrm{m}, \mathrm{H}=15 \mu \mathrm{m}, \mathrm{P}=75 \mu \mathrm{m}$

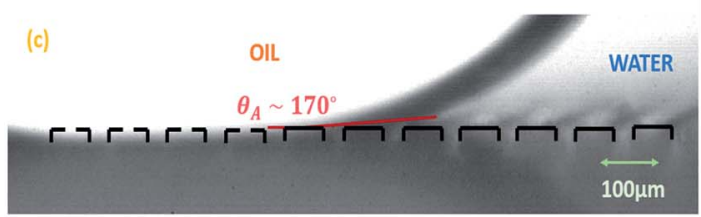

$D=50 \mu \mathrm{m}, \mathrm{H}=15 \mu \mathrm{m}, \mathrm{P}=100 \mu \mathrm{m}$ ii. Receding Contact Angle

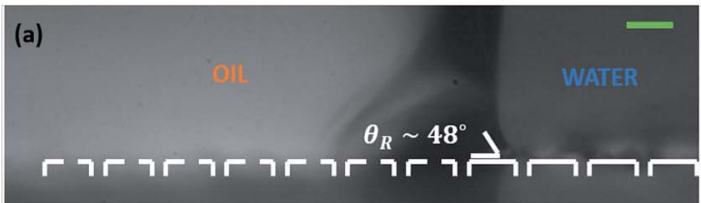

$\mathrm{D}=50 \mu \mathrm{m}, \mathrm{H}=15 \mu \mathrm{m}, \mathrm{P}=60 \mu \mathrm{m}$

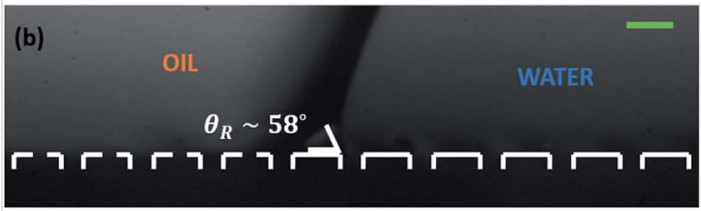

$\mathrm{D}=50 \mu \mathrm{m}, \mathrm{H}=15 \mu \mathrm{m}, \mathrm{P}=75 \mu \mathrm{m}$

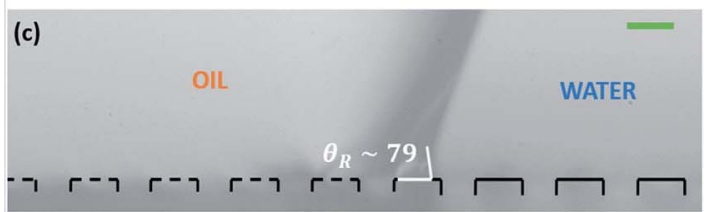

$\mathrm{D}=\mathbf{5 0} \mu \mathrm{m}, \mathrm{H}=15 \mu \mathrm{m}, \mathrm{P}=100 \mu \mathrm{m}$

Fig. 5 (i) Advancing contact angles of laser oil drops for the different micro-patterned substrates kept underwater: (a) $D=50 \mu \mathrm{m}, H=15 \mu \mathrm{m}, P=$ $60 \mu \mathrm{m}$, (b) $D=50 \mu \mathrm{m}, H=15 \mu \mathrm{m}, P=75 \mu \mathrm{m}$ and (c) $D=50 \mu \mathrm{m}, H=15 \mu \mathrm{m}, P=100 \mu \mathrm{m}$. The micro-pillars have been drawn for clarity. It should be noted that the scale bars for (a)-(c) are different as indicated in sub-figures. (ii) Receding contact angles of laser oil drops for the different micropatterned substrates kept underwater: (a) $D=50 \mu \mathrm{m}, H=15 \mu \mathrm{m}, P=60 \mu \mathrm{m}$, (b) $D=50 \mu \mathrm{m}, H=15 \mu \mathrm{m}, P=75 \mu \mathrm{m}$ and (c) $D=50 \mu \mathrm{m}, H=15 \mu \mathrm{m}$, $P=100 \mu \mathrm{m}$. The micro-pillars have been drawn for clarity. The scale bar represents $50 \mu \mathrm{m}$.

(a)

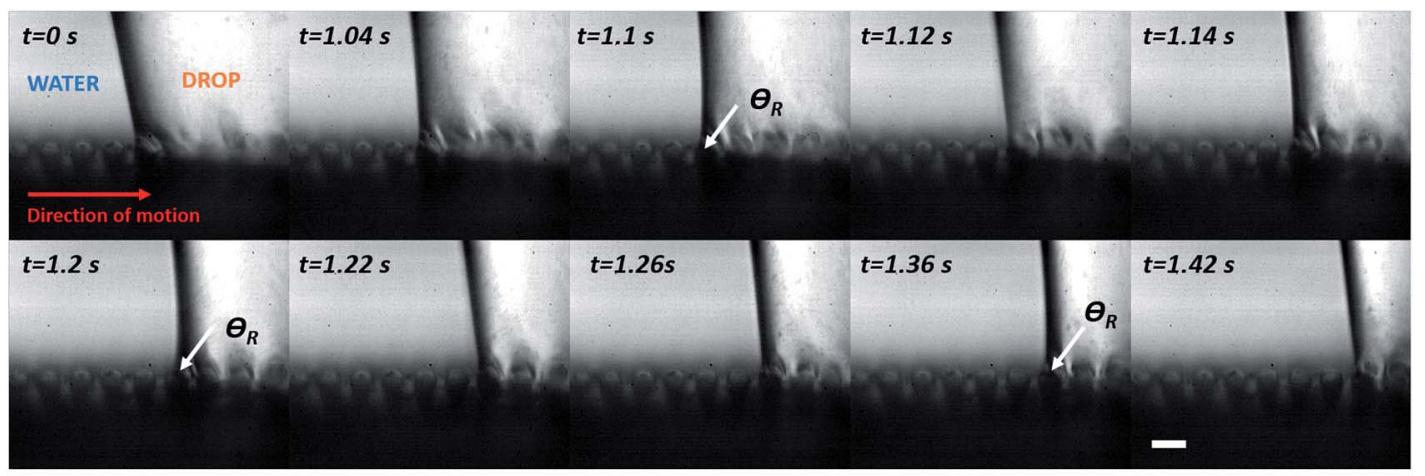

(b)

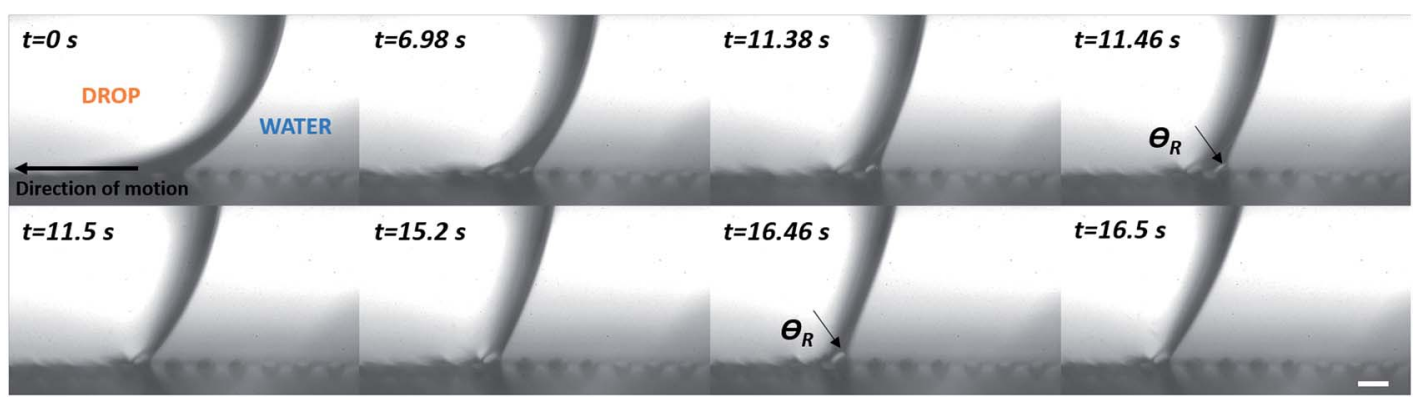

Fig. 6 (a) Time snaps of the receding motion of a laser oil drop on the micro-patterned substrate with pitch $75 \mu \mathrm{m}(D=50 \mu \mathrm{m}$ and $H=15 \mu \mathrm{m})$. It can be seen that while receding, the three phase contact line shifted from one pillar top to the adjacent one, one at a time. The frames corresponding to $t=1.1 \mathrm{~s}$ and $t=1.36 \mathrm{~s}$ show the three phase contact line just before a shift to the adjacent pillar top and indicates the receding contact angle, $\theta_{\mathrm{R}}=58^{\circ}$, in this case. The scale bar represents $100 \mu \mathrm{m}$. (b) Time snaps of the receding motion of a laser oil drop on the micropatterned substrate with pitch $100 \mu \mathrm{m}(D=50 \mu \mathrm{m}$ and $H=15 \mu \mathrm{m})$. Here also, while receding, the three phase contact line shifted from one pillar top to the adjacent one, one at a time. The frames corresponding to $t=11.46 \mathrm{~s}$ and $t=16.46 \mathrm{~s}$ show the three phase contact line just before a shift to the adjacent pillar top and indicates the receding contact angle, $\theta_{\mathrm{R}}=79^{\circ}$, in this case. The scale bar represents $100 \mu \mathrm{m}$. $t=0$ represents an arbitrary moment during receding and does not represent equilibrium configuration. 


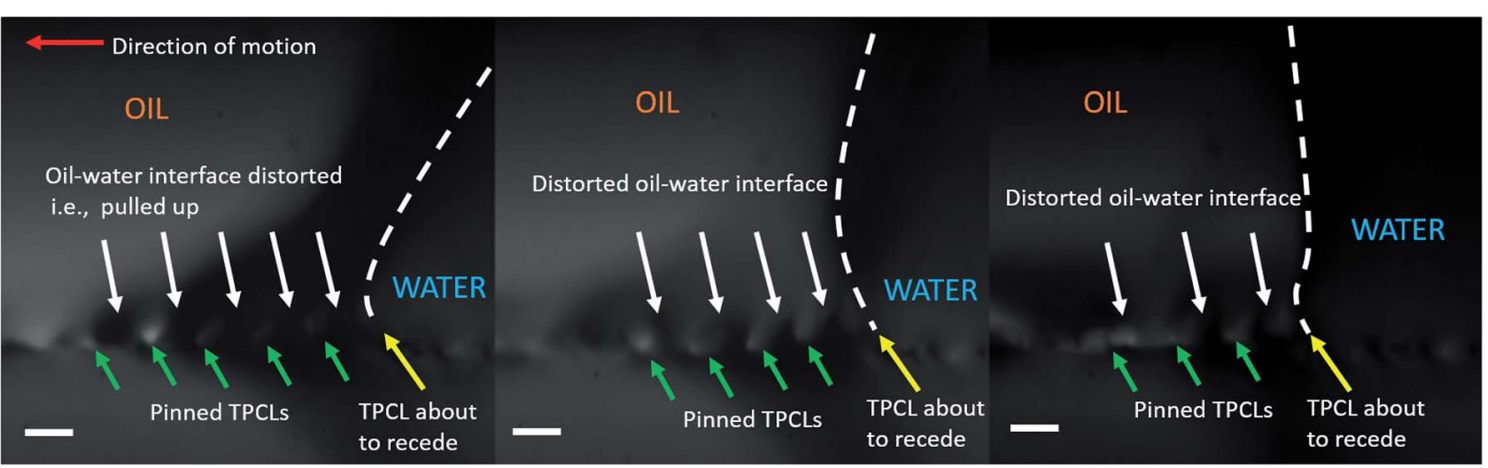

Fig. 7 Receding dynamics of an oil drop on an under-water micro-patterned substrate with pillar pitch $75 \mu$ m performed with a slight change in camera-lens orientation for the purpose of visualization of the receding mechanism in all the three axes. The pillars, not drawn for this case, are located beneath the three-phase contact lines (TPCLs). When oil is pumped out of the drop, the oil-water interface connecting two adjacent TPCLs gets distorted (represented by the white arrows), essentially gets pulled up while the TPCLs remain pinned on their respective pillar tops (green arrows). The receding motion then takes place with one TPCL receding at a time (yellow arrow) while the other adjacent TPCLs remain pinned on their respective pillar tops. The dashed line represents the oil-water interface corresponding to the TPCL which is receding. The scale bar represents $50 \mu \mathrm{m}$.

(i.e., inviscid) medium, the advancing contact angle is always close to $180^{\circ}$, which is obvious since the three-phase contact line needs to reach such an angle to touch the next set of pillars. On the other hand, the receding contact angle values varied significantly with pillar pitch, as shown in Fig. 5(ii). The increase in receding contact angles with increasing pillar pitch is also commonly found in hysteresis studies in air medium. $^{14,20,68}$ However, the wide spread in hysteresis for under-liquid substrates obtained here is something new and warrants further study.

It is also important to understand the motion of the threephase contact line during advancing and receding motions of the oil drop over the pillars of varying pitch. When liquid was withdrawn from the drop during the course of receding, it was found that for the smallest pillar pitch (i.e., $60 \mu \mathrm{m}$ ), the threephase contact line receded a number of pillars at once (see ESI video S1 $\dagger$ ). However, as shown in Fig. 6, for the larger pillar pitches (i.e., $75 \mu \mathrm{m}$ and $100 \mu \mathrm{m}$ ) the contact line was observed to jump individual pillars while receding (see Fig. 6(a) and (b)). Still, it was not clear whether the drop was in a Wenzel or Cassie-Baxter state. For underwater systems, the problem lies in conventional imaging of drop-substrate system since it usually provides a clear visualization of the three phase contact line (TPCL) only in two axes ( $x$ and $z$, in our case). It does not provides a clear picture of the neighboring TPCLs or the dropwater interface between two adjacent TPCLs. Hence, to ascertain whether the drop was in a Wenzel or Cassie-Baxter state, it was necessary to image the receding mechanism with a clear visualization of the receding TPCL and the neighboring dropwater interface behavior. Such visualization is normally performed under a microscope for static drop configurations. However for dynamic configurations, i.e., a receding drop, observing the process with camera-lens system is more suitable.

To achieve a visualization of the drop receding motion where all the three axes are visible, images were captured by positioning the camera at an angle of about 10 from the reference plane used earlier. Interestingly, it was found that while receding, for patterned substrate with pitch $75 \mu \mathrm{m}$, locally the drop-water interface is pulled up while the TPCL remained pinned on the adjacent pillar tops (see Fig. 7). Then, a single TPCL receded at a time while the neighboring TPCLs remained pinned. Similar phenomenon was also observed for the substrate with pillar pitch $60 \mu \mathrm{m}$, but due to the contact line jumping over a number of pillars at once, such motion could not be effectively captured. The pulling up of the drop-water interface while receding, strongly indicates that the wetting for those two substrates is not a Wenzel configuration. Rather it is either a metastable Cassie-Baxter or a mixed wetting state with partial filling of the space between the pillars. ${ }^{69}$

\section{Discussions}

\subsection{Static configuration}

Table 3 reports the comparison of the experimentally observed advancing and receding contact angles for the different underwater micro-patterned substrates with those predicted by Wenzel and Cassie-Baxter equations, i.e., by substituting $\theta_{\mathrm{eq}}$ with $\theta_{\mathrm{OW}, \mathrm{A}}$ and $\theta_{\mathrm{OW}, \mathrm{R}}$ in eqn (3) and (4), where $\theta_{\mathrm{OW}, \mathrm{A}}$ and $\theta_{\mathrm{OW}, \mathrm{R}}$ are the experimentally observed advancing and receding contact angles on the corresponding underwater flat reference substrate. This approach is also a major part of this entire debate revolving around the applicability of using these theories to predict hysteresis behavior. ${ }^{14,17,19-21,41,42}$ Nevertheless, we use this approach to compare our experimentally observed values. It can be seen from Table 3, except for the receding contact angle for the micro-patterned substrate with pitch $100 \mu \mathrm{m}$, none of the advancing and receding angles comply with those predicted by either Wenzel or Cassie-Baxter theory. This indicates that a Wenzel configuration observed in air medium does not always necessarily translate to Wenzel configuration for underwater systems. In the present case, since the substrate is underwater, the surrounding pressure is not atmospheric but with the added contribution of the hydrostatic head, $p_{\mathrm{H}}\left(\sim \rho_{\mathrm{W}} g h\right)$ posed by the height $h$ of water column present in the cuvette. Therefore eqn 
Table 3 Comparison of advancing and receding contact angles of oil on underwater micro-patterned surfaces with those predicted by Wenzel (eqn (3)) and Cassie-Baxter (eqn (4)) configurations (i.e., by computing $\theta_{\text {Wenzel }}$ and $\theta_{\mathrm{CB}}$ from eqn (3) and (4), respectively, using experimentally observed advancing and receding contact angles $\theta_{\mathrm{OW}, \mathrm{A}}$ and $\left.\theta_{\mathrm{OW}, \mathrm{R}}\right)$

\begin{tabular}{llll}
\hline Pitch $(\mu \mathrm{m})$ & $\begin{array}{l}\text { Observed advancing and } \\
\text { receding CAs } \theta_{\mathrm{A}} / \theta_{\mathrm{R}}\end{array}$ & $\begin{array}{l}\text { Theoretical advancing and } \\
\text { receding CAs (Wenzel) } \theta_{\mathrm{A}} / \theta_{\mathrm{R}}\end{array}$ & $\begin{array}{l}\text { Theoretical advancing and } \\
\text { receding CAs }(\mathrm{CB}) \theta_{\mathrm{A}} / \theta_{\mathrm{R}}\end{array}$ \\
\hline 0 (flat) & $103^{\circ}\left(\theta_{\mathrm{OW}, \mathrm{A}}\right) \pm 2^{\circ} / 80^{\circ}\left(\theta_{\mathrm{OW}, \mathrm{R}}\right) \pm 2^{\circ}$ & - & - \\
60 & $175^{\circ} \pm 2^{\circ} / 48^{\circ} \pm 2^{\circ}$ & $112^{\circ} / 73^{\circ}$ & $125^{\circ} / 111^{\circ}$ \\
75 & $171^{\circ} \pm 2^{\circ} / 58^{\circ} \pm 2^{\circ}$ & $108^{\circ} / 76^{\circ}$ & $136^{\circ} / 126^{\circ}$ \\
100 & $170^{\circ} \pm 2^{\circ} / 79^{\circ} \pm 2^{\circ}$ & $106^{\circ} / 78^{\circ}$ & $147^{\circ} / 140^{\circ}$
\end{tabular}

(1), applicable for substrates kept in air, needs to be modified to take into account this hydrostatic head. Hence, the condition for the critical transition pressure for an under-liquid substrate can be written as:

$$
p_{\mathrm{D}}-\left(p_{\mathrm{A}}+p_{\mathrm{H}}\right)>-\frac{2 \gamma_{\mathrm{OW}} \cos \theta_{\mathrm{OW}}}{(P-D)}
$$

We argue that since the pressure difference across the dropwater interface below the suspended drop between two adjacent pillars, $p_{\mathrm{D}}-\left(p_{\mathrm{A}}+p_{\mathrm{H}}\right)$ is significantly lower than the corresponding scenario in air, i.e., $p_{\mathrm{D}}-p_{\mathrm{A}}$, for the substrates with relatively smaller pitches (i.e., $60 \mu \mathrm{m}$ and $75 \mu \mathrm{m}$ ), the critical transition pressure is never reached resulting in a Cassie-Baxter or an intermediate configuration. It is to be noted that existence of such critical pressure for superrepellancy in air for wetting liquids on re-entrant structures has been discussed by Liu and Kim. ${ }^{6}$ For the substrate with the relatively larger pitch, i.e., 100 $\mu \mathrm{m}$, it appears that the critical transition pressure was achieved and a Wenzel configuration was realized, evident from its receding contact angle value. It should be noted here that the height of the water column ( $\sim 15-18 \mathrm{~mm}$ for the present study) cannot be varied as an experimental parameter in terms of order of magnitude. This is due to challenges in our current contact angle measurement system as well as in other commercially available systems, in terms of the vertical needle movement and the drop size requirement, restricting such a variation. A more comprehensive computational study of the critical pressure transition criterion would have been helpful in throwing more light into this complex phenomenon of energy barrier mediated wetting configuration. However, such a study is beyond the scope of the present work and can be treated as a topic for future investigation.

\subsection{Receding contact angle values}

From the observation of receding motion, even though a metastable Cassie-Baxter state (or even a mixed wetting state) can be inferred for the drop on the underwater substrates with pitches $60 \mu \mathrm{m}$ and $75 \mu \mathrm{m}$, the receding contact angle values exhibited on these substrates appears to be puzzling. A wide hysteresis is indicative of a Wenzel behavior. But our visualization of the receding phenomenon (see Fig. 7) clearly shows a receding mechanism unlikely of a Wenzel-like configuration. Often, the edge effect ${ }^{70,71}$ on such pillar structures provides results which deviates from the conventional Wenzel and Cassie-Baxter states. However, no such edge effects were noticed in our visualization of either the equilibrium shape and the three phase contact line movement during advancing and receding motion of the drop. For the low contact angles observed, it can be due to the fact that a thin layer of water may have been left behind when the three phase contact line receded, as pointed out by Patankar et al. ${ }^{13}$ in a similar study carried out for substrates in air medium. Another possibility can be a precursor film formation, ${ }^{72,73}$ which better explains such curvature close to the three-phase contact line while receding. This is presently not well understood and needs to be investigated further in terms of the dynamics of liquid-liquid displacement. In our recent study related to under-liquid drop coalescence, ${ }^{74}$ we have delineated the role of the surrounding denser and viscous medium (i.e., water). Commonly observed wetting phenomena like spreading ${ }^{73,75,76}$ and coalescence of sessile drops $^{74}$ are very different for under-liquid substrates than substrates in ambient air where parameters like the surrounding liquid density and viscosity play key roles in dictating the dynamics of how one dense liquid displaces another. Hence, new studies are required with significant modification to the governing theories that hold true for air medium by taking into account the role of the surrounding viscous medium, rather than a mere extrapolation of these theories. Also, recent advancement in experimental techniques like laser scanning confocal microscopy ${ }^{77}$ would be of great help to understand such under-liquid wetting behavior.

\section{Conclusion}

The present study reports a first of its kind detailed investigation of wetting characteristics of oil drops on underwater micropatterned substrates. For this purpose, we have performed wetting studies of three different micro-pillared substrates, where each substrate has same pillar height and diameter but varying pillar pitch of $60 \mu \mathrm{m}, 75 \mu \mathrm{m}$, and $100 \mu \mathrm{m}$. It was found that for such cases, the wetting characteristics (in terms of static, advancing and receding contact angles) cannot be formulated using the conventional framework of Wenzel ${ }^{10}$ and Cassie-Baxter ${ }^{11}$ theories. It is clear that the conventional theories as well as trends seen for wetting on micro-patterned substrates kept in air medium ${ }^{1,2,8,14-16,18,37-42}$ do not necessarily translate to similar behavior on under-liquid systems. The static drop configuration for such an underwater substrate was found to conform to a metastable Cassie-Baxter or a mixed wetting state evident from the study of the receding mechanism of the 
oil drop in underwater system. The energy barrier associated with displacing a surrounding dense medium and filling up the space between the pillars should be accounted for to predict which configuration (Wenzel, Cassie-Baxter, metastable Cassie-Baxter or a mixed wetting state) is thermodynamically favorable. Also, since an outer liquid medium possess significant viscous effects, the dynamics of liquid-liquid displacement needs to be considered to understand the behavior of the threephase contact line in such systems which is crucial for appropriate characterization of the contact angle, particularly the receding contact angle. With growing number of underwater applications as well as a need to mitigate environmental effects of major oil spills, which has become a growing concern in the wake of the DeepWater Horizon oil spill event by $\mathrm{BP},{ }^{78}$ this basic study on understanding wettability of underwater micropatterned substrates garners relevance in designing appropriate oil repellant and corrosion resistant surfaces. The results presented here points to our lack of comprehensive theoretical understanding of the mechanisms that dictate wetting of under-liquid micro-patterned substrates and begs the need to have a new theoretical framework for such systems.

\section{Acknowledgements}

The authors would like to thank Natural Sciences and Engineering Research Council of Canada for their financial support through Grant \# RGPIN-2014-05236 and RTI-2015-472734.

\section{References}

$1 \mathrm{M}$. Nosonovsky and B. Bhushan, Multiscale dissipative mechanisms and hierarchical surfaces: friction, superhydrophobicity, and biomimetics, Springer-Verlag, Heidelberg, Germany, 2008.

2 J. Genzer and K. Efimenko, Biofouling, 2006, 22, 339-360.

3 Y. Zheng, X. Gao and L. Jiang, Soft Matter, 2007, 3, 178-182.

$4 \mathrm{M}$. Nosonovsky and B. Bhushan, Curr. Opin. Colloid Interface Sci., 2009, 14, 270-280.

5 Y. Liu and G. Li, J. Colloid Interface Sci., 2012, 388, 235-242.

6 T. Liu and C. Kim, Science, 2014, 346, 6213.

7 P. R. Waghmare, S. Mitra, N. S. K. Gunda and S. K. Mitra, RSC Adv., 2015, 5, 82374-82380.

8 B. Bhushan, Y. C. Jung and K. Koch, Philos. Trans. R. Soc., A, 2009, 367, 1631-1672.

9 S. Martin and B. Bhushan, J. Colloid Interface Sci., 2016, 474, 206-215.

10 R. N. Wenzel, Ind. Eng. Chem., 1936, 28, 988-994.

11 A. Cassie and S. Baxter, Trans. Faraday Soc., 1944, 40, 546551.

12 L. Gao and T. J. McCarthy, Langmuir, 2007, 23, 3762-3765.

13 N. A. Patankar, Langmuir, 2003, 19, 1249-1253.

14 C. Dorrer and J. Rühe, Langmuir, 2006, 22, 7652-7657.

15 G. McHale, Langmuir, 2007, 23, 8200-8205.

16 M. Nosonovsky, Langmuir, 2007, 23, 9919-9920.

17 C. Extrand, Langmuir, 2002, 18, 7991-7999.

18 A. Marmur, Langmuir, 2003, 19, 8343-8348.
19 C. Priest, T. W. Albrecht, R. Sedev and J. Ralston, Langmuir, 2009, 25, 5655-5660.

20 D. Öner and T. J. McCarthy, Langmuir, 2000, 16, 7777-7782.

21 C. Extrand, Langmuir, 2003, 19, 3793-3796.

22 L. Gao and T. J. McCarthy, Langmuir, 2007, 23, 13243.

23 L. Gao and T. J. McCarthy, Langmuir, 2009, 25, 14105-14115.

24 F. Bartell and J. Shepard, J. Phys. Chem., 1953, 57, 455-458.

25 J. Joanny and P.-G. De Gennes, J. Chem. Phys., 1984, 81, 552562.

26 W. Choi, A. Tuteja, J. M. Mabry, R. E. Cohen and G. H. McKinley, J. Colloid Interface Sci., 2009, 339, 208-216.

27 N. Anantharaju, M. V. Panchagnula, S. Vedantam, S. Neti and S. Tatic-Lucic, Langmuir, 2007, 23, 11673-11676.

28 R. Raj, R. Enright, Y. Zhu, S. Adera and E. N. Wang, Langmuir, 2012, 28, 15777-15788.

29 S. T. Larsen and R. Taboryski, Langmuir, 2009, 25, 12821284.

30 A. Gauthier, M. Rivetti, J. Teisseire and E. Barthel, Phys. Rev. Lett., 2013, 110, 046101.

31 W. Li and A. Amirfazli, J. Colloid Interface Sci., 2005, 292, 195-201.

32 T. L. Liu, Z. Chen and C.-J. Kim, Soft Matter, 2015, 11, 15891596.

33 A. Shastry, S. Abbasi, A. Epilepsia and K. F. Bohringer, in International Solid-State Sensors, Actuators and Microsystems Conference (Transducers'07), 2007, pp. 599-602.

34 R. Dufour, M. Harnois, V. Thomy, R. Boukherroub and V. Senez, Soft Matter, 2011, 7, 9380-9387.

35 T. Wu and Y. Suzuki, Sens. Actuators, B, 2011, 156, 401-409. 36 E. Bormashenko, J. Colloid Interface Sci., 2011, 360, 317-319. 37 J. Bico, C. Tordeux and D. Quéré, Europhys. Lett., 2001, 55, 214.

38 N. A. Patankar, Langmuir, 2004, 20, 8209-8213.

39 B. Bhushan and Y. C. Jung, Ultramicroscopy, 2007, 107, 10331041.

40 B. He, N. A. Patankar and J. Lee, Langmuir, 2003, 19, 49995003.

41 K.-Y. Yeh, L.-J. Chen and J.-Y. Chang, Langmuir, 2008, 24, 245-251.

42 Y. Kwon, S. Choi, N. Anantharaju, J. Lee, M. Panchagnula and N. Patankar, Langmuir, 2010, 26, 17528-17531.

43 C. Frankiewicz and D. Attinger, Nanoscale, 2016, 8, 39823990.

44 J. Bico, C. Marzolin and D. Quéré, Europhys. Lett., 1999, 47, 220. 45 Z. Yoshimitsu, A. Nakajima, T. Watanabe and K. Hashimoto, Langmuir, 2002, 18, 5818-5822.

46 R. E. Johnson Jr and R. H. Dettre, J. Phys. Chem., 1964, 68, 1744-1750.

47 P. Forsberg, F. Nikolajeff and M. Karlsson, Soft Matter, 2011, 7, 104-109.

48 N. A. Patankar, Langmuir, 2010, 26, 8941-8945.

49 A. Giacomello, M. Chinappi, S. Meloni and C. M. Casciola, Phys. Rev. Lett., 2012, 109, 226102.

50 Q.-S. Zheng, Y. Yu and Z.-H. Zhao, Langmuir, 2005, 21, 12207-12212.

51 H. Kusumaatmaja, M. Blow, A. Dupuis and J. Yeomans, Europhys. Lett., 2008, 81, 36003. 
52 E. Bormashenko, Philos. Trans. R. Soc., A, 2010, 368, 4695-4711. 53 B. Liu and F. F. Lange, J. Colloid Interface Sci., 2006, 298, 899909.

54 E. Bormashenko, Adv. Colloid Interface Sci., 2015, 222, 92103.

55 Y. C. Jung and B. Bhushan, Langmuir, 2009, 25, 14165-14173.

56 V. Hejazi and M. Nosonovsky, Langmuir, 2011, 28, 21732180.

57 I. Palamà, S. D'Amone, V. Arcadio, D. Caschera, R. Toro, G. Gigli and B. Cortese, J. Mater. Chem. A, 2015, 3, 3854-3861.

58 M. Jin, S. Li, J. Wang, Z. Xue, M. Liao and S. Wang, Chem. Commun., 2012, 48, 11745-11747.

59 Q. Cheng, M. Li, Y. Zheng, B. Su, S. Wang and L. Jiang, Soft Matter, 2011, 7, 5948-5951.

60 M. Nosonovsky and B. Bhushan, Philos. Trans. R. Soc., A, 2009, 367, 1511-1539.

61 B. Bhushan, Beilstein J. Nanotechnol., 2011, 2, 66-84.

62 V. Hejazi, A. E. Nyong, P. K. Rohatgi and M. Nosonovsky, Adv. Mater., 2012, 24, 5963-5966.

63 N. S. K. Gunda, M. Singh, Y. Purwar, S. L. Shah, K. Kaur and S. K. Mitra, Biomed. Microdevices, 2013, 15, 959-971.

64 N. S. K. Gunda, J. Joseph, A. Tamayol, M. Akbari and S. K. Mitra, Microfluid. Nanofluid., 2013, 14, 711-721.

65 N. S. K. Gunda, B. Bera, N. K. Karadimitriou, S. K. Mitra and S. M. Hassanizadeh, Lab Chip, 2011, 11, 3785-3792.
66 P. R. Waghmare, S. Das and S. K. Mitra, Soft Matter, 2013, 9, 7437-7447.

67 P. R. Waghmare and S. K. Mitra, Langmuir, 2010, 26, 1708217089.

68 H. Kusumaatmaja and J. Yeomans, Langmuir, 2007, 23, 6019-6032.

69 S. Farsinezhad, P. R. Waghmare, B. D. Wiltshire, H. Sharma, S. Amiri, S. K. Mitra and K. Shankar, RSC Adv., 2014, 4, 33587-33598.

70 F.-M. Chang, S.-J. Hong, Y.-J. Sheng and H.-K. Tsao, J. Phys. Chem. C, 2010, 114, 1615-1621.

71 F.-M. Chang, Y.-J. Sheng and H.-K. Tsao, Appl. Phys. Lett., 2009, 95, 204107.

72 P.-G. De Gennes, Rev. Mod. Phys., 1985, 57, 827.

73 D. Bonn, J. Eggers, J. Indekeu, J. Meunier and E. Rolley, Rev. Mod. Phys., 2009, 81, 739.

74 S. Mitra and S. K. Mitra, Phys. Rev. E: Stat., Nonlinear, Soft Matter Phys., 2015, 92, 033013.

75 K. Chaudhury and S. Chakraborty, Langmuir, 2015, 31, 41694175.

76 S. Mitra and S. K. Mitra, Langmuir, 2016, 32, 8843-8848.

77 F. Schellenberger, N. Encinas, D. Vollmer and H.-J. Butt, Phys. Rev. Lett., 2016, 116, 096101.

78 A. C. Ortmann, J. Anders, N. Shelton, L. Gong, A. G. Moss and R. H. Condon, PLoS One, 2012, 7, e42548. 